\title{
SÍNTESE E CARACTERIZAÇÃo DE ZEÓLITAS OBTIDAS A PARTIR DE CINZAS VOLANTES DE CARVÃO
}

\author{
Luciane Ventirini Lacerda $^{{ }^{*}}$, Felipe Amorim Berutti ${ }^{1}$, Annelise Kopp Alves ${ }^{1}$ \\ 1 Programa de Pós-graduação em Engenharia de Minas, Metalúrgica e de Materiais (PPGE3M), Universidade Federal do Rio \\ Grande do Sul, 90035-190, Porto Alegre-RS, Brasil.
}

*E-mail: eng.luventurini@gmail.com

\section{RESUMO}

Usinas termoelétricas a carvão geram aproximadamente 3 milhões de toneladas de cinzas ao ano. Estas cinzas podem receber uma aplicação nobre como, por exemplo, fotocatalisadores aplicados à descontaminação de águas poluídas por corantes da indústria têxtil. Isso é possível através de utilização das cinzas como fonte de silício para a síntese de zeólitas. Desta forma, neste trabalho foram sintetizadas zeólitas do tipo sodalita, utilizando-se cinzas volantes de carvão da região de Candiota-RS, com diferentes teores de alumínio, e na presença de $\mathrm{NaOH}$ como alcalinizante, variando-se o tempo de envelhecimento. Os produtos formados foram caracterizados por DRX. A área superficial foi determinada por isotermas de adsorção de $\mathrm{N}_{2}$ (BET) e sua morfologia foi analisada por MEV e MET. A atividade fotocatalítica foi medida através do acompanhamento da degradação de solução contendo o corante azul de metileno. Os resultados indicaram que a razão $\mathrm{Si}: \mathrm{Al}$ e o tempo de envelhecimento influenciam significativamente as propriedades da zeólita sintetizada. A zeólita obtida é do tipo sodalita, formada por aglomerados com partículas primárias de $100 \mathrm{~nm}$, com área superficial de aproximadamente $6 \mathrm{~m}^{2} / \mathrm{g}$. A atividade fotocatalítica foi bastante significativa e foram observados indicativos de propriedades adsorventes.

Palavras-chave: zeólita, sodalita, cinzas volantes de carvão, fotocatálise.

\section{Introdução}

Foi em 1756 que a história das zeólitas iniciou quando o mineralogista sueco Axel Fredrick Cronstedt notou que um determinado mineral natural quando aquecido na chama liberava bruscamente vapor de água [1,2]. Esse mineral quando se resfriava voltava a se hidratar, de forma que poderia ser exposto a sucessivos ciclos de hidratação-desidratação. Atualmente, a definição para o termo zeólitas sugere que qualquer material que apresente estrutura tridimensional composta por átomos tetraedricamente coordenados, unidos entre si por átomos de oxigênio, pode ser considerado zeólita [1].

As zeólitas normalmente são sintetizadas a partir de condições hidrotermais, consistindo na cristalização de um composto a partir de uma substância nutriente (amorfa ou cristalina), na presença de água e de um agente mineralizante. A síntese normalmente ocorre em temperaturas na faixa de $25^{\circ} \mathrm{C}$ a $300^{\circ} \mathrm{C}$ e sua natureza é definida por fatores cinéticos. É possível sintetizar zeólitas de diferentes estruturas ou ainda a mesma zeólita com diferentes composições químicas, fazendo variar a composição das soluções de síntese e as condições operacionais [5]. A cinética do processo de cristalização sofre influência por qualquer variável que faça modificar o meio reacional. Entre os mais importantes parâmetros, destacam-se: tempo [3,6], temperatura [3,6], agitação da mistura reacional [1], adição de sementes [7], alcalinidade do sistema de cristalização [6,1], diluição do sistema de cristalização [8,9], razão Si/Al [3] e cátions inorgânicos [9].

As usinas termelétricas que usam carvão como combustível são responsáveis pela geração significativa de resíduos sólidos (cinzas). De acordo com Kalkreuth [10], no Brasil sete usinas termoelétricas dos Estados do RS e de SC produzem aproximadamente 3 milhões de toneladas de cinzas a cada ano, as quais são compostas de 65 a $85 \%$ de cinza leve e 15 a $35 \%$ de cinza pesada. Como apenas uma pequena parcela de cinzas é aproveitada na produção de clínquer para a fabricação de cimento Portland, o restante é disposto em bacias de cinzas ou utilizado para tapar poços de minas esgotados [10].

De outro lado, a indústria têxtil encontra-se entre os 8 setores mais importantes da atividade industrial brasileira, sendo que nas regiões Sul e Sudeste encontram-se $80 \%$ destas indústrias. Do ponto de vista ambiental, a remoção da cor dos efluentes é uma das dificuldades enfrentadas por este setor. $\mathrm{Na}$ 
busca de novas tecnologias para resolver o problema, a fotocatálise aparece como uma alternativa tecnológica importante, principalmente quando utilizados catalisadores de baixo custo e alta atividade catalítica.

Neste contexto, o intuito deste trabalho foi estudar a influência dos parâmetros de síntese de zeólitas a partir de cinzas de carvão. Foram investigados a concentração $\mathrm{NaOH}$ (empregado como agente alcalinizante), a razão Si:Al e o tempo de envelhecimento, e sua influência nas propriedades físicas e químicas das estruturas formadas, bem como suas propriedades fotocatalíticas.

\section{Metodologia}

Primeiramente, as cinzas volantes do carvão foram secas em estufa a uma temperatura de $150^{\circ} \mathrm{C}$ por $24 \mathrm{~h}$. A seguir, deu-se início à síntese das zeólitas, pelo processo hidrotérmico em meio alcalino. A metodologia utilizada foi adaptada a partir do método descrito pela IZA (International Zeolite Association) [11]. A Figura 1 mostra um fluxograma da metodologia empregada na síntese das zeólitas investigadas neste trabalho.

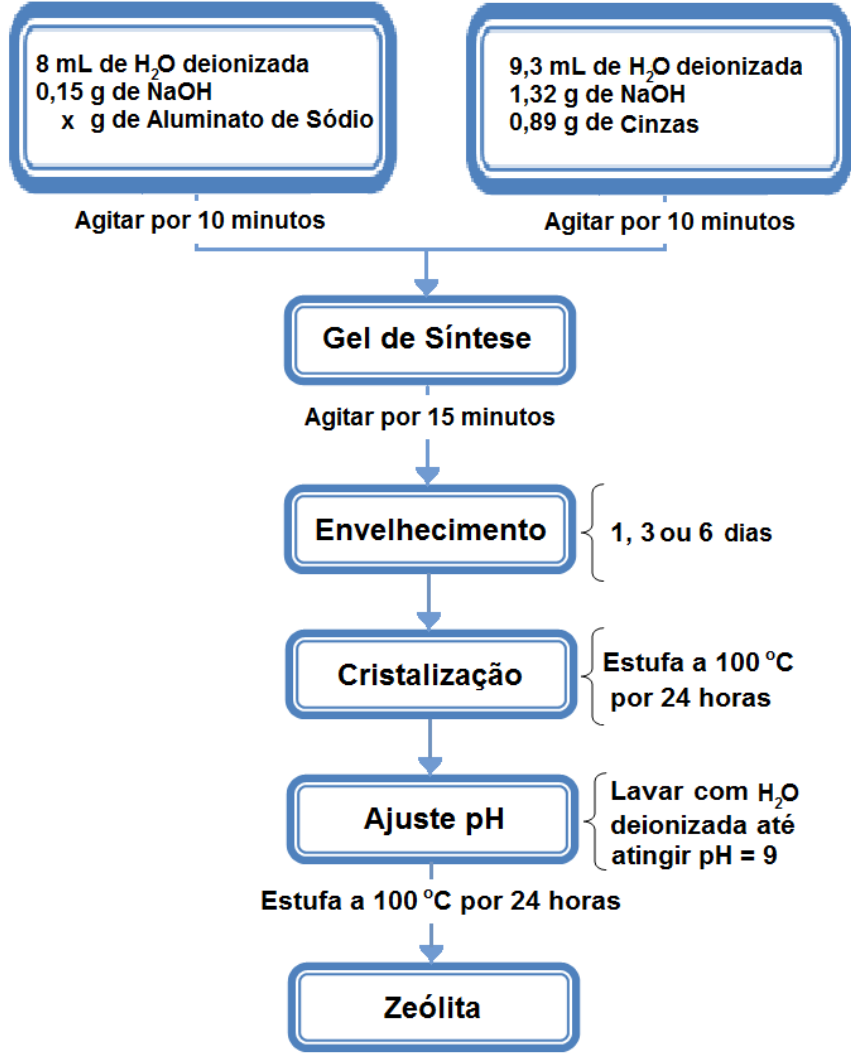

Figura 1 - Fluxograma da metodologia empregada na síntese das zeólitas investigadas neste trabalho.
Uma solução base de $\mathrm{NaOH}$ (Sigma-Aldrich) foi preparada com duas concentrações diferentes: $0,23 \mathrm{~mol} / \mathrm{L}$ e 3,5 $\mathrm{mol} / \mathrm{L}$, e a partir dela outras duas foram feitas. A primeira continha $\mathrm{NaOH} 3,35 \mathrm{~mol} / \mathrm{L}$ e $0,89 \mathrm{~g}$ de cinzas (como fonte de $\mathrm{SiO}_{2}$ ). A segunda solução continha $\mathrm{NaOH} 0,23 \mathrm{~mol} / \mathrm{L}$ e aluminato de sódio. Este último foi adicionado em diferentes quantidades a fim de resultar as razões Si:Al de $1: 1 ; 2: 1 ; 3: 1 \mathrm{e}$ 4:1. Essas soluções foram homogeneizadas com o auxílio de um agitador magnético durante 10 minutos à temperatura ambiente. Para a formação do gel de síntese, as soluções foram misturadas e homogeneizadas por mais 15 minutos, também à temperatura ambiente. $\mathrm{O}$ gel sofreu um processo de envelhecimento por 1, 3 ou 6 dias à temperatura ambiente. Após, seguiu-se o processo de cristalização que ocorreu em estufa a $110^{\circ} \mathrm{C}$ por 24 horas. $\mathrm{O}$ material sólido assim formado foi lavado com água deionizada até atingir o $\mathrm{pH} 9$ e seco em estufa a $110^{\circ} \mathrm{C}$ por 24 horas. Após este processo, as amostras foram cominuídas com auxílio de gral e pistilo. $\mathrm{O}$ processo foi repetido para solução base $\mathrm{NaOH} \mathrm{3,5}$ $\mathrm{mol} / \mathrm{L}$, sendo que a primeira solução continha $\mathrm{NaOH} 6,15 \mathrm{~mol} / \mathrm{L}$ e a segunda $\mathrm{NaOH} 3,5 \mathrm{~mol} / \mathrm{L}$.

A caracterização mineralógica consistiu em determinar as fases formadas a partir dos dados da difração de raios X (DRX), utilizando um difratômetro de raios $\mathrm{X}$ (Philips, modelo X'pert MPD) equipado com um monocromador de grafite e ânodo rotativo de cobre, operando a $40 \mathrm{kV}$ e $40 \mathrm{~mA}$. A área superficial específica foi determinada por adsorção de nitrogênio (NOVA 1000e, Quanta-Chrome Instruments), utilizando a técnica de Brunauer, Emmett e Teller (BET). A morfologia dos materiais sintetizados foi analisada por meio de microscopia eletrônica de varredura (MEV - JEOL, modelo JSM 6060) e de transmissão (MET - JEOL, modelo JEM 1200 Exll).

As amostras também foram avaliadas quanto a sua atividade fotocatalítica. Em um ensaio fotocatalítico típico, foi utilizado um corante (azul de metileno) cuja descoloração foi observada através da espectroscopia UV-Vis. Uma dispersão foi preparada com $125 \mathrm{~mL}$ de uma solução contendo $20 \mathrm{ppm}$ do corante e $50 \mathrm{mg}$ da amostra. Esta mistura foi homogeneizada com o auxílio de ultrassom, em uma câmara escura por 15 minutos, para melhor dispersão do material e adsorção inicial do corante sobre a superfície do catalisador. Uma amostra de aproximadamente $4 \mathrm{~mL}$ foi retirada logo após a homogeneização, sendo então a mistura transferida para o reator de vidro. Após o sistema de iluminação UVA $(\lambda=365 \mathrm{~nm})$ ser ligado, amostras de $4 \mathrm{~mL}$ foram coletadas a cada 5 minutos e colocadas em cuvetas de polimetilmetacrilato (PMMA) com caminho óptico de $10 \mathrm{~mm}$ para posterior análise da absorbância por espectrofotometria. 


\section{Resultados e discussões}

\subsection{Composição mineralógica e área superficial}

As Figuras 2 e 3 apresentam a análise da composição mineralógica realizada por difração de raios $\mathrm{X}$ das amostras com diferentes concentrações de $\mathrm{NaOH}$ na solução base. Observa-se que o produto zeolítico formado foi identificado como sendo do tipo sodalita. O quartzo e a mulita também identificados são provenientes das cinzas de carvão que não foram completamente convertidos durante o tratamento proposto [13]. A formação de mulita na cinza está relacionada com a combustão do carvão mineral a temperaturas acima de $1050^{\circ} \mathrm{C}$, sendo que o carvão mineral que originou a cinza utilizada neste trabalho é queimado a $1400^{\circ} \mathrm{C}$. Sabe-se que a mulita é considerada como um composto de grande dificuldade de dissolução durante a síntese hidrotérmica [6], contudo observou-se a presença de mulita apenas nas amostras com maior teor de $\mathrm{NaOH}$ na solução base (3,5 $\mathrm{mol} / \mathrm{L})$.

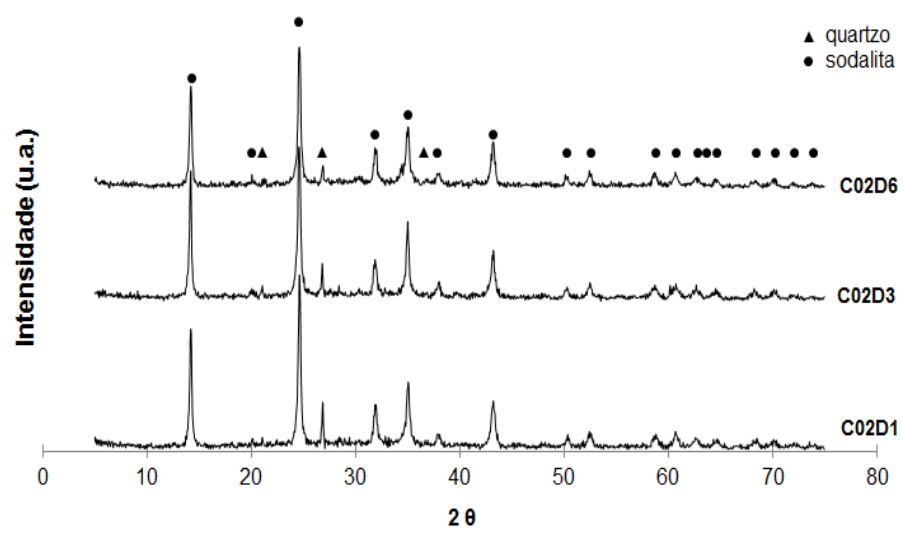

Figura 2. Difratogramas das zeólitas sintetizadas com solução contendo 0,23 $\mathrm{mol} / \mathrm{L}$ de $\mathrm{NaOH}$ envelhecidas 1,3 ou 6 dias.

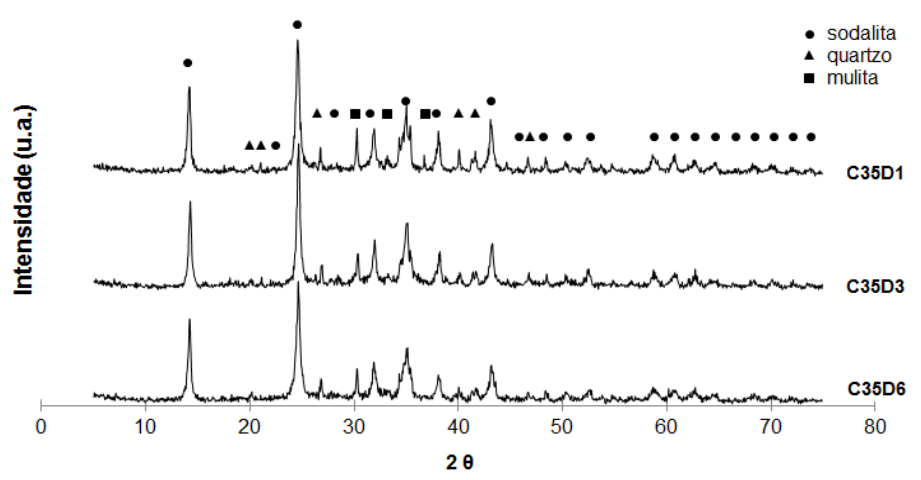

Figura 3 - Difratogramas das zeólitas sintetizadas com solução contendo 3,5 $\mathrm{mol} / \mathrm{L}$ de $\mathrm{NaOH}$ envelhecidas 1,3 ou 6 dias.
Pela análise mineralógica, também não foi possível constatar uma diferença significativa na composição mineralógica ou cristalinidade ao se comparar os diferentes tempos de envelhecimentos destas matrizes. Contudo, aparentemente a fase cristalina quartzo perde intensidade no espectro de DRX com um maior tempo de envelhecimento. Estes comportamentos podem ser justificados pelo fato de que o processo de envelhecimento ocorreu em temperatura ambiente e logo, a energia fornecida durante esta fase não foi suficiente para ocasionar a transformação completa da zeólita.

Em se tratando da relação entre a razão $\mathrm{Si}: \mathrm{Al}$ utilizadas e a área superficial específica dos pós sintetizados, observa-se que entre 1 e 3 dias de envelhecimento não houve mudança significativa na área superficial específica para cada uma das razões estudadas. Contudo, após 6 dias de envelhecimento, todas as amostras apresentaram área superficial específica com valores semelhantes, em torno de $4,0 \mathrm{~m}^{2} / \mathrm{g}$. Como referência, indicou-se na Figura 4 o valor da área superficial específica da cinza utilizada $\left(3,4 \mathrm{~m}^{2} / \mathrm{g}\right)$.

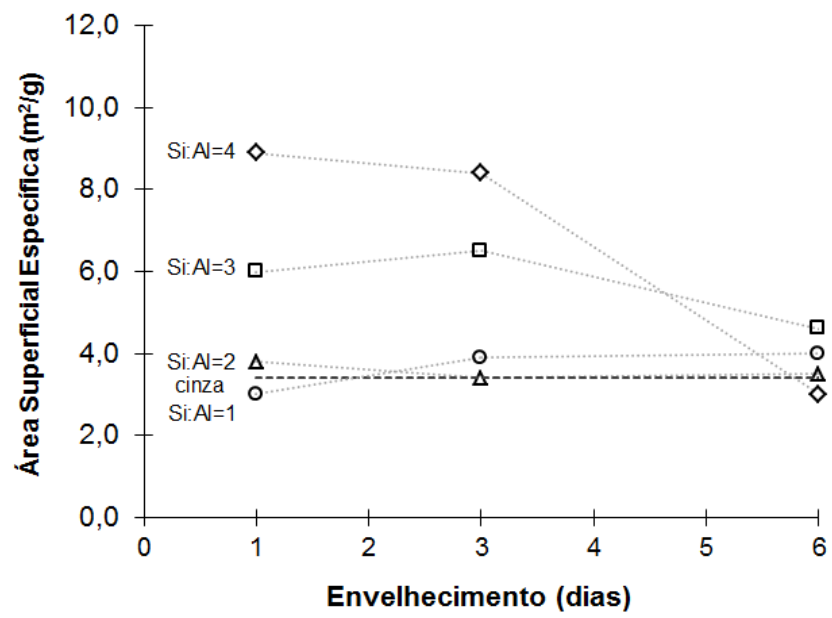

Figura 4. Área superficial específica das amostras com diferentes razões Si:Al em função do tempo de envelhecimento.

As análises mineralógicas das amostras sintetizadas com diferentes razões $\mathrm{Si}: \mathrm{Al}$ em relação ao tempo de envelhecimento (1 a 6 dias) podem ser observadas nas Figuras 5 e 6 . Nota-se, especialmente para as amostras com razão $\mathrm{Si}: \mathrm{Al}=4$, a presença das fases quartzo, mulita e muscovita, provenientes provavelmente da conversão incompleta das cinzas de carvão [12], além da zeólita tipo sodalita, com intensidade mais significativa para a amostra envelhecida por 6 dias. Destaca-se a presença de quartzo como fase majoritária após 1 e 3 dias de envelhecimento para todas as razões $\mathrm{Si}: \mathrm{Al}$, com exceção da razão 
1:1, onde a sodalita é a fase majoritária para todos os tempos de envelhecimento.

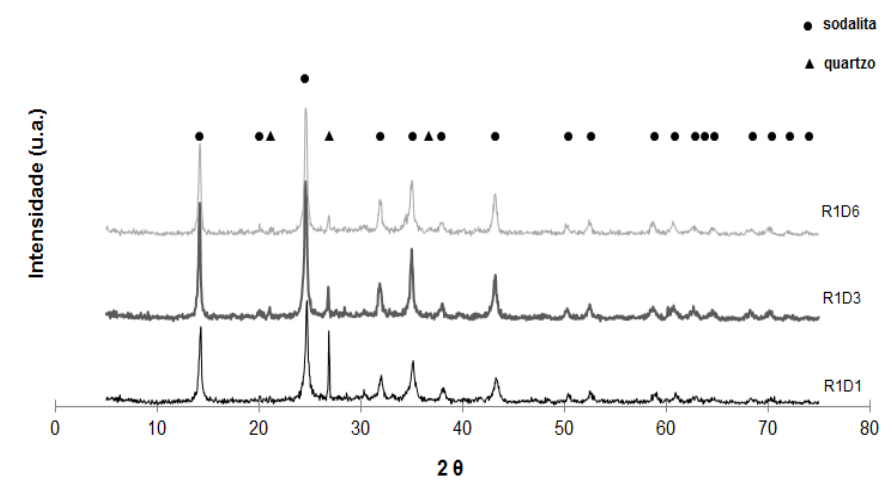

Figura 5 - Difratogramas das zeólitas sintetizadas utilizando a razão $\mathrm{Si}: \mathrm{Al}=1$, envelhecidas 1,3 ou 6 dias.

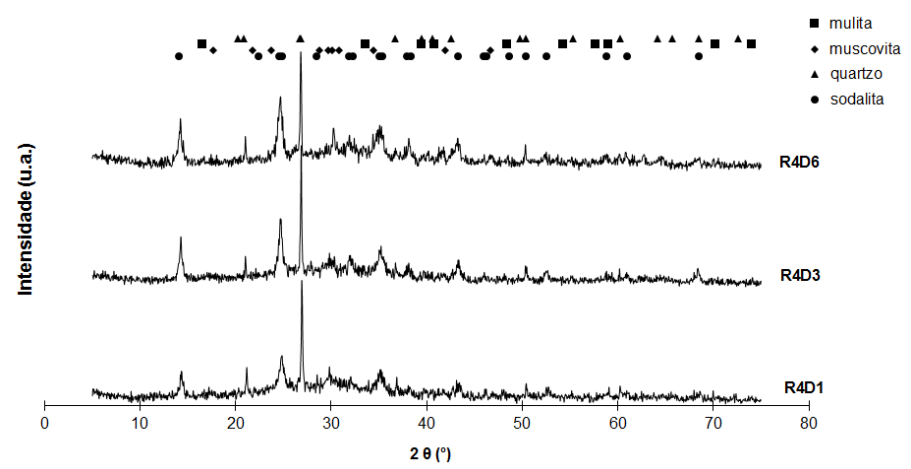

Figura 6 - Difratogramas das zeólitas sintetizadas utilizando a razão $\mathrm{Si}: \mathrm{Al}=4$, envelhecidas 1,3 ou 6 dias.

O aumento da razão $\mathrm{Si}: \mathrm{Al}$ dificultou a síntese da sodalita, pois o aumento da quantidade de silício nas amostras favoreceu a presença de quartzo como fase predominante. Baseado nos dados apresentados, há indicativos de que o silício aumenta o tempo necessário para a cristalização da sodalita. Este fato condiz com relatos que afirmam que a formação das estruturas zeolíticas ocorre devido à progressiva diminuição da razão Si:Al com o tempo, ou seja, há o desaparecimento de estruturas com alta razão $\mathrm{Si}: \mathrm{Al}$, onde o quartzo é fase majoritária, e o aumento ou até mesmo o surgimento de estruturas com menor razão Si:Al (estruturas mais estáveis) [6].

\subsection{Morfologia}

As imagens por microscopia eletrônica de varredura $(\mathrm{MEV})$ apresentadas na Figura 7 permitem observar a morfologia do material sintetizado após 6 dias de envelhecimento e com diferentes razões $\mathrm{Si}$ :Al. As zeólitas sintetizadas apresentam-se como aglomerados de partículas arredondadas e de tamanhos variados. A superfície irregular pode ser decorrente da presença de cristais de zeólita que se precipitaram sobre as partículas das cinzas de carvão durante o tratamento proposto. Os cristalitos menores podem ser resultado da presença de impurezas nas cinzas que leva a uma nucleação rápida, prejudicando o crescimento do cristal [13].

As imagens obtidas por microscopia eletrônica de transmissão (MET) para as amostras zeolíticas sintetizadas com razão $\mathrm{Si}: \mathrm{Al}$ 3:1 e 4:1 após 6 ou 1 dia de envelhecimento, respectivamente, são apresentadas na Figura 8. É possível observar aglomerados com tamanhos da ordem de $100 \mathrm{~nm}$, sendo estes formados por cristais ainda menores. Foram observados pontos esféricos nos cristais das amostras que provavelmente são pontos de nucleação sem tempo e/ou temperatura suficientes para crescer e cristalizar.
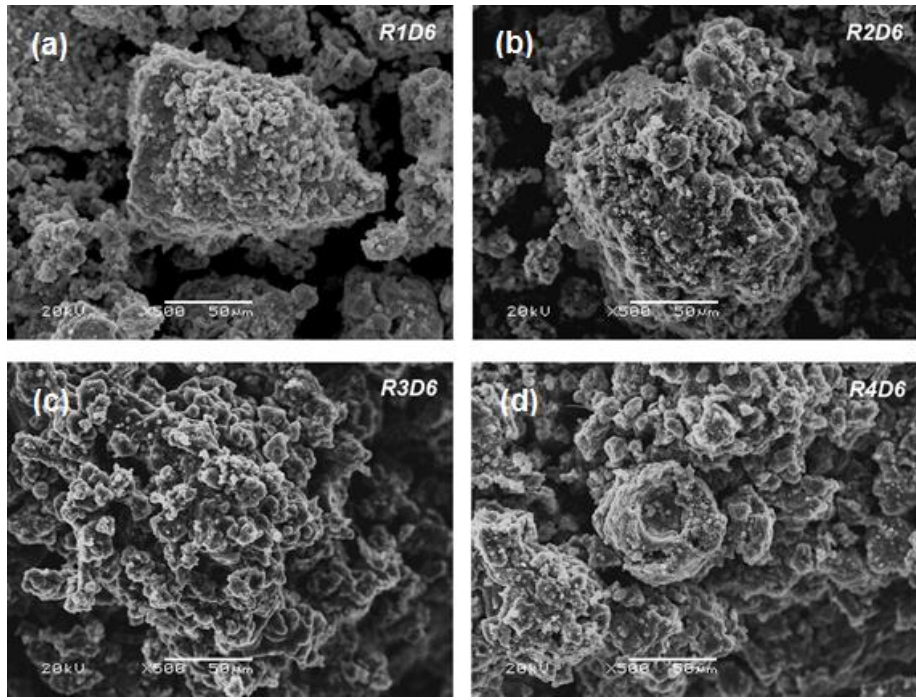

Figura 7 - Imagens por MEV dos materiais sintetizados após 6 dias de envelhecimento utilizando razões Si:Al a)1:1, b) 2:1 c) 3:1 d) 4:1.

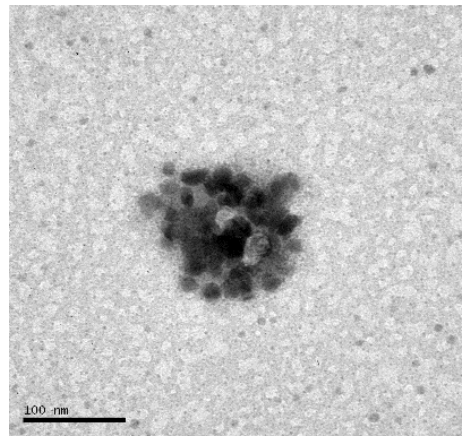

(a)

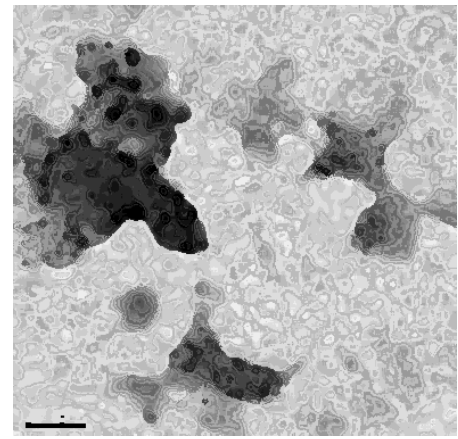

(b)
Figura 8. Imagens obtidas por MET dos materiais sintetizados: a) após 6 dias de envelhecimento utilizando razão $\mathrm{Si}: \mathrm{Al}=3: 1 ; \mathrm{b}$ ) após 1 dia de envelhecimento utilizando razão $\mathrm{Si}: \mathrm{Al}=4: 1$. 


\subsection{Atividade fotocatalítica}

A Figura 9 apresenta a atividade fotocatalítica das amostras sintetizadas, utilizando diferentes razões $\mathrm{Si}: \mathrm{Al}$ e tempos de envelhecimento, em função da cinética de degradação de uma solução do corante azul de metileno.

Observa-se que após uma hora de irradiação as zeólitas sintetizadas degradaram entre 62 e $86 \%$ do corante utilizado. O catalisador obtido, utilizando-se a razão 2:1 $\mathrm{Si}: \mathrm{Al}$ e seis dias de envelhecimento (R2D6), foi aquele que apresentou uma considerável atividade fotocatalítica se comparado com os demais, degradando aproximadamente $86 \%$ do corante em uma hora de irradiação UV.

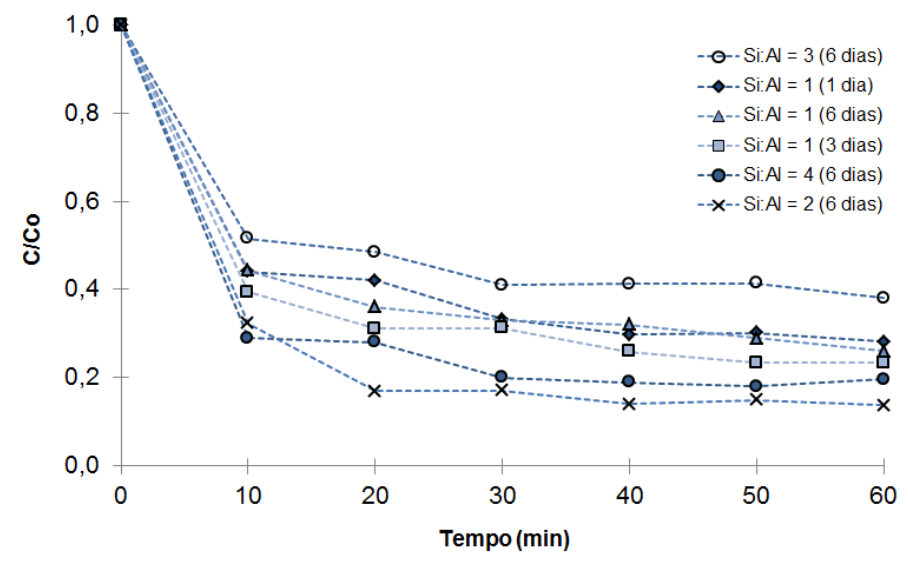

Figura 9. Variação da concentração do corante azul de metileno por irradiação UV em presença das amostras sintetizadas utilizando diferentes razões Si:Al e tempos de envelhecimento.

Comparando-se com os resultados de DRX, observa-se que esta amostra contém aparentemente maior fração de sodalita que as demais amostras, considerando a intensidade relativa dos picos de difração. É interessante observar também, que após os primeiros 10 minutos há uma queda acentuada na concentração do corante e após este período a concentração se mantém aparentemente constante. Isto pode ser um indicativo de que o material sintetizado pode atuar também como um adsorvente de moléculas corantes.

\section{Conclusões}

Foi possível obter zeólitas do tipo sodalita, utilizando-se cinzas volantes de carvão como fonte de silício. A análise por difração de raios $\mathrm{X}$ identificou a formação majoritária da fase sodalita, além de quartzo, mulita e muscovita, estas últimas originalmente presentes na cinza e não convertidas completamente. A área superficial específica e as fases formadas foram afetadas pela razão Si:Al. Observou-se que quanto menor a razão Si:Al utilizada na síntese, maior a conversão em sodalita. As imagens por micrografias eletrônicas de varredura mostraram a formação de aglomerados de superfície rugosa. Por micrografia eletrônica de transmissão, foi possível identificar a partículas com ordem de tamanho de cerca de $100 \mathrm{~nm}$. Os materiais sintetizados possuem atividade fotocatalítica significativa, degradando até $86 \%$ do corante empregado em 60 minutos. Destaca-se também a possível característica adsorvente deste material.

\section{Agradecimentos}

Os autores agradecem à CAPES (Coordenação de Aperfeiçoamento de Pessoal de Nível Superior) e ao CNPQ (Conselho Nacional de Desenvolvimento Científico e Tecnológico) pelo auxílio financeiro.

\section{SYNTHESIS AND CHARACTERIZATION OF ZEOLITES FROM COAL FLY ASH}

\begin{abstract}
Coal power plants generate about 3 million tons of ash per year. This ash can receive a noble application, for example, as photocatalyst applied to the decontamination of waters polluted with textile industry dyes. This is possible through use of the ash as a silicon source for zeolite synthesis. Thus, this work investigated the synthesis of sodalite type zeolite using coal fly ash in the presence of different aluminum contents, $\mathrm{NaOH}$ as an alkalizing agent and as function of aging time. The products were characterized by $\mathrm{XRD}, \mathrm{N}_{2}$ adsorption isotherms (BET) and their morphologies were examined by SEM and TEM. The photocatalytic activity was measured by monitoring the degradation of a methylene blue solution. The results shown that $\mathrm{Si}$ Al ratio and the aging time significantly influences the properties of the synthesized zeolite. The zeolite type sodalite was constituted of agglomerated with primary particles size of $100 \mathrm{~nm}$, with a specific surface area of ca. $6 \mathrm{~m}^{2} / \mathrm{g}$. The photocatalytic activity was significant and indicatives of adsorbent properties were observed.
\end{abstract}

Keywords: Zeolite, sodalite, coal fly ash, photocatalysis.

\section{Referências}

[1] MARTÍNEZ, J. G.; PARIENTE, J. P.; - Materiales Zeolíticos: Síntesis, Propiedades y Aplicaciones. Publicaciones Universidad de Alicante, 2002. p.13. apud Petkowicz, D. I. Zeólitas sintetizadas com fontes alternativas de silício e alumínio. Aplicação em fotocatálise. Dissertação (Mestrado), Programa de 
Pós-Graduação em Ciência dos Materiais, Universidade Federal do Rio Grande do Sul, 2009.

[2] MASCARENHAS, A. J. S.; OLIVEIRA, E. C.; PASTORE, H. O.; - Peneiras Moleculares: Selecionando as Moléculas por seu Tamanho. In: Cadernos Temáticos de Química Nova na Escola. Edição Especial, maio/2001.

[3] PETKOWICZ, D. I. - Zeólitas sintetizadas com fontes alternativas de silício e alumínio. Aplicação em fotocatálise. Dissertação (Mestrado), Programa de Pós-Graduação em Ciência dos Materiais, Universidade Federal do Rio Grande do Sul, 2009.

[4] DA LUZ, A. B. - Zeólitas: propriedades e usos industriais. Série Tecnologia Mineral, Rio de Janeiro: CETEM/CNPq, 1995.

[5] SIMÕES, A. N.; NEIVA, L. S.; ARAÚJO, A.P.; OLIVEIRA, J. B. L.; RODRIGUES, M. G. F.; GAMA, L.; - Estudo da influência dos parâmetros de síntese na obtenção de zeólitas. Revista Eletrônica de Materiais e Processos, Vol. 6.1, p. 35-38, 2011.

[6] FERRET, S. L. - Zeólitas de cinzas de carvão: Síntese e Uso. Tese (Doutorado), Programa de Pós-Graduação em Engenharia de Minas, Metalúrgica e de Materiais (PPGE3M), Universidade Federal do Rio Grande do Sul, 2004.

[7] MOOR, P.E.A.; BEELEN, T.P.M.; SANTEM, R.A.; J. Chem. Phys. B, In situ Observation of nucleation and cristal growth in zeolite synthesis. A small-angle X-ray scattering investigation on Si-TPA-MFI, Vol. 103, 1639, 1999.

[8] IWASAKI, A.; KUDO, I.; SANO, T.; In. Progress in zeolite and microporous materials. Studies in surface science and catalysis; Chon, H.; Ihm, S-K (Edt.). Amsterdam: Elsevier, 1997.

[9] SZOSTAK, R. - Molecular Sieves - Principles oh synthesis and identification; Van Nostrand Reinhold: New York, 1989.

[10] KALKREUTH, W.; HOLTZ, M.; KERN, M.; MACHADO, G.; MEXIAS, A.; SILVA, M. B.; WILLETT, J.; FINKELMAN, R.; BURGER, H. Petrology and chemistry of Permian coals from the Paraná Basin: 1. Santa Terezinha, Leão -Butiá and Candiota coal fields, Rio Grande do Sul, Brasil. International Journal of Coal Geology, v. 68, p. 79-119, 2006.

[11] Site oficial The International Zeolite Association. Disponível em: http://www.iza-online.org. Acesso em 19/09/2015.

[12] IZIDORO, J. C.; - Estudo sobre remoção de íons metálicos em água usando zeólitas sintetizadas a partir de cinzas de carvão. Dissertação (Mestrado), Instituto de pesquisas energéticas e nucleares, Autarquia associada à Universidade de São Paulo, 2008.
[13] FUNGARO, D. A.; BORRELY, S. I.; Síntese e caracterização de zeólitas de cinzas de carvão modificada por surfactante catiônico. Revista Cerâmica, Vol. 58, p. 77-83, 2012. 